\title{
NGC 5128 - a nearby laboratory for planetary nebulae in a giant early-type galaxy
}

\author{
Jeremy R. Walsh ${ }^{1}$, George H. Jacoby ${ }^{2}$, Harald Kuntschner ${ }^{1}$, \\ Reynier F. Peletier ${ }^{3}$, Marina Rejkuba ${ }^{1}$, Nicholas A. Walton ${ }^{4}$, and \\ Kristin A. Woodley ${ }^{5}$ \\ ${ }^{1}$ European Southern Observatory, Garching, Germany \\ ${ }^{2}$ Giant Magellan Telescope Corporation / Carnegie Observatories, Pasadena, USA; \\ ${ }^{3}$ Kapteyn Astronomical Institute, Groningen, the Netherlands; \\ ${ }^{4}$ Institute for Astronomy, Cambridge, United Kingdom; \\ ${ }^{5}$ Department of Physics \& Astronomy, University of British Columbia, Canada
}

\begin{abstract}
NGC 5128 at $3.8 \mathrm{Mpc}$ is the nearest large elliptical galaxy and is ideally suited to a detailed study of its planetary nebula population. Two spectroscopic programmes are summarised. More than 1200 PNe candidates are known from imaging campaigns in NGC 5128 and accurate radial velocities of 1070 have been measured with the VLT FLAMES/Giraffe spectrometer. From these data a variety of studies of the galaxy kinematics are enabled, such as search for PN sub-groups, representing the relics of accretion of small galaxies. Emission line spectra were observed with VLT FORS and the light element abundances determined for 40 $\mathrm{PNe}$ through photoionization modelling. A spread in $\mathrm{O}$ abundance of about 0.9 dex is found but no obvious radial gradient out to $19 \mathrm{kpc}$. Comparison of the $\mathrm{O}$ abundance from these PN with the metallicity for the stellar population in the neighbourhood of the PN will probe the star formation and enrichment history of the galaxy. Full results from this analysis will be presented in a forthcoming paper.
\end{abstract}

Keywords. Planetary nebulae: general, galaxies: elliptical, galaxies: individual (NGC 5128), techniques: spectroscopic

\section{NGC 5128 and its PNe}

At a distance of $3.8 \mathrm{Mpc}$ (distance modulus 27.91: Harris et al. 2010), NGC 5128 is the nearest large/giant elliptical galaxy (Hubble type S0p; $L_{V}=-21.5 \mathrm{mag}$.). It is a unique galaxy in the local environment with a dust lane and H II regions in its centre indicating a recent infall event (Graham 1979), an active galactic nucleus shrouding a super-massive black hole (Neumayer 2010), with X-ray (Kraft et al. 2002) and $\gamma$-ray detections (Steinle 2010), as well as shells (Malin et al.1983) and streams (Peng et al. 2002) in its outer periphery; see Isreal (1998) for a review and an update in the special issue of PASA Vol. 27, 2010. However NGC 5128 is actually a rather typical early-type galaxy, as up to $75 \%$ have emission lines or other indicators of dust and molecular gas, as shown by the Sauron and ATLAS ${ }^{3 D}$ surveys, e.g. Sarzi et al. (2006). It also has many planetary nebulae $(\mathrm{PNe})$.

The PNe at the distance of NGC 5128 are point sources (size $<0.05^{\prime \prime}$ ) which are easily detectable from on/off band [O III] $5007 \AA$ imaging. Currently $>1200$ candidates have been detected (Hui et al. 1993; Peng et al. 2004; Walsh et al. in prep). The brightest $\mathrm{PN}$ has $m_{5007 A}=23.5 \mathrm{mag}$. and $\mathrm{PNe}$ are detectable to $\geqslant 27.5 \mathrm{mag}$, except in the high 

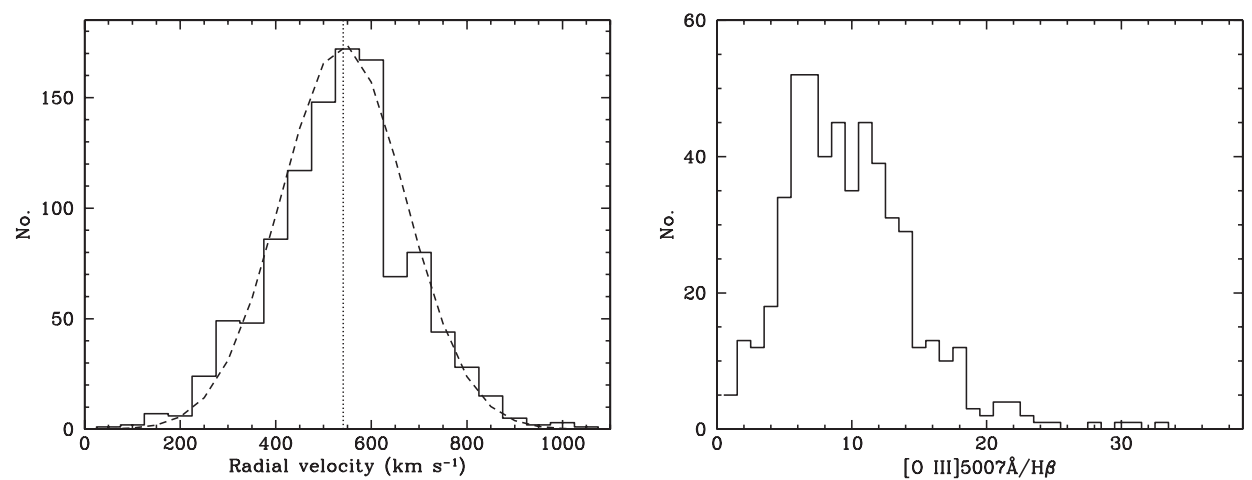

Figure 1. (a): histogram of $1176 \mathrm{PN}$ radial velocities in NGC 5128 from FLAMES Medusa spectra. The mean velocity $\left(541 \mathrm{kms}^{-1}\right)$ and a single Gaussian fit $\left(\sigma=130 \mathrm{kms}^{-1}\right)$ to the distribution are shown. (b): histogram of the [O III] $5007 / \mathrm{H} \beta$ ratio for $529 \mathrm{PN}$ in NGC 5128.

surface brightness nuclear region. Despite their faintness, they can serve as probes of the metallicity state of the ISM, the star formation history and the kinematics of the luminous and ultimately dark matter.

In this paper a summary is presented of optical spectroscopy of 51 of the brighter PN yielding line fluxes of $\mathrm{He}, \mathrm{N}, \mathrm{O}, \mathrm{Ne}, \mathrm{S}, \mathrm{Ar}$ emission lines and higher resolution spectra for a much larger sample of high precision radial velocities, all with the ESO VLT.

\section{Multi-object observations}

\subsection{Radial velocities}

Spectra with the FLAMES Giraffe spectrograph in Medusa mode (MOS with field of view $27^{\prime}, 1321.2^{\prime \prime}$ fibres) at a resolving power of of 7500 were observed of $534 \mathrm{PN}$ from the catalogue of Hui et al. (1993). With the ESO NTT and EMMI instrument an imaging search in [O III] and off-line was conducted over a large area of the galaxy to catalogue more PNe. Including the imaging of Hui et al. (1993) and Peng et al. (2004), 1260 unique PN candidates have been detected in NGC 5128. Further FLAMES spectra of many of these PNe were made, resulting in radial velocities from fits to the detected emission lines for at total of 1176 PNe in NGC 5128 with typical velocity errors of $3 \mathrm{kms}^{-1}$. Since the range for the LR2 grating setting of the FLAMES Medusa spectrograph is 4600-5100A, including $\mathrm{He}$ II, $\mathrm{H} \beta$ and the [O III] doublet, these spectra also provide a large sample of line ratios e.g. $[\mathrm{O} \mathrm{III]} / \mathrm{H} \beta$. Figure 1a shows a histogram of the collected radial velocities and Figure $1 \mathrm{~b}$ the histogram of the $[\mathrm{O}$ III $] 5007 \AA / \mathrm{H} \beta$ ratio of the $529 \mathrm{PNe}$ with both lines detected. Full details will appear in Rejkuba et al. (in prep.).

\subsection{Spectrophotometry}

The FORS spectrometer was used in its multislit mode with 19 slitlets up to $20^{\prime \prime}$ in length for low resolution $(\mathrm{R} \sim 1500)$ spectroscopy in 3 fields at radial offsets of $4^{\prime}$ (F42), 7.5' (F56) and 14.5' (F34); field designation is from Hui et al. (1993). Catalogued PN were

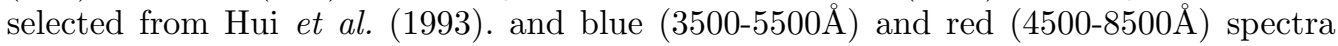
were obtained covering many of the important optical diagnostic lines with exposures up to $3 \mathrm{hrs}$ per field per spectral range. Some of the slitlets have multiple PN, including some not in the Hui et al. (1993) catalogue, and a total of 51 spectra were extracted $(23.5<$ $\left.m_{5007 A}<27.5\right)$, including 9 in the outermost field. For about $80 \%$ of the PN spectra, lines of at least $\mathrm{H} \beta, \mathrm{H} \alpha,[\mathrm{O} \mathrm{III}]$, and [N II] were detected. The weak, electron temperature 

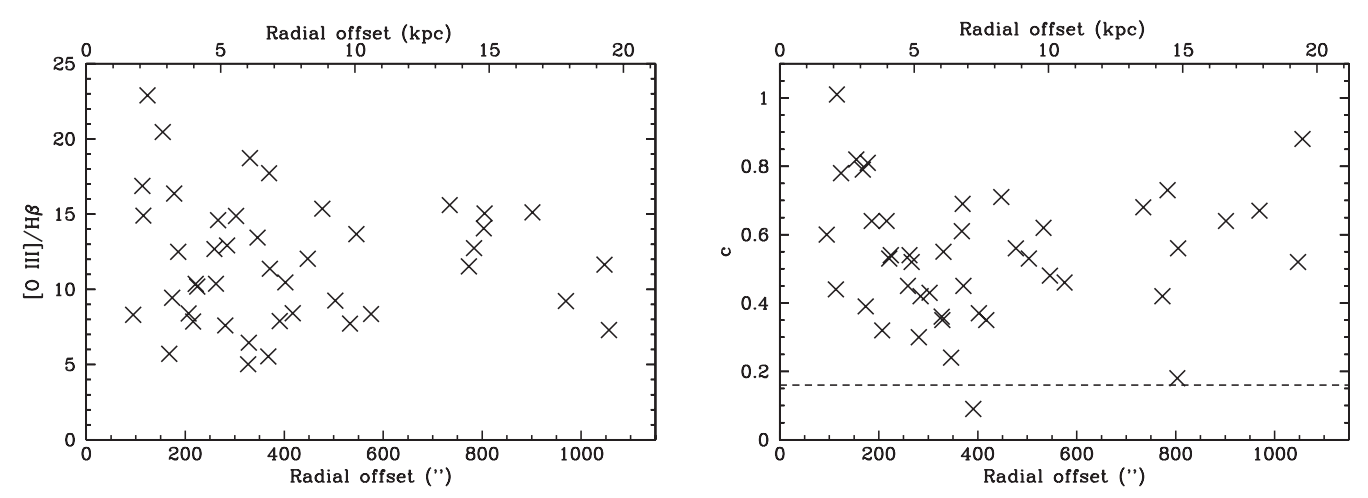

Figure 2. (a): variation of the observed $[\mathrm{O}$ III $] 5007 \AA / \mathrm{H} \beta$ ratio with radial distance based on VLT FORS spectrometry. (b): variation of the logarithmic extinction at $\mathrm{H} \beta$, c, measured from the $\mathrm{H} \alpha / \mathrm{H} \beta$ ratio with radial distance for $42 \mathrm{PNe}$ in NGC 5128. The dashed line at shows the Milky Way line-of-sight extinction in this direction.

diagnostic line [O III] $4363 \AA$ was detected in $10 \mathrm{PN}$ (at low signal-to-noise, $\leqslant 4$ ) and the density sensitive ratio $[\mathrm{S}$ II $] 6716 / 6731 \AA$ in 7 PN. Fig. $2 a$ shows the $[\mathrm{O}$ III $] 5007 \AA / \mathrm{H} \beta$ ratio v. galactocentric distance. The full results will appear in Walsh et al. in prep.

\section{Selected results}

\subsection{Kinematics}

The PNe velocities, supplemented with the 563 globular cluster (GC) velocities (e.g. Woodley et al. (2010) and references therein) provide an unprecedented set of test particles for galaxy scale kinematic modelling (c.f. Cortesi et al. (2011)). The positional information and accurate velocities provide a database to search for sub-groups of $\mathrm{PNe}$ (and GCs) that can provide a signature of possible dwarf galaxy accretion events. Woodley \& Harris (2011) found several possible (4 GC and 4 PN) such groups based on the velocities of Hui et al. (1995) and Peng et al. (2004). With the FLAMES data, groups PNe 2 and 4 survive with the same set of criteria (viz. subgroup linking length $2^{\prime}$ and velocity difference $20 \mathrm{~km} \mathrm{~s}^{-1}$ ). An independent analysis of the FLAMES dataset yielded the tightest grouping of $5 \mathrm{PN}$ within a diameter of $50^{\prime \prime}(900 \mathrm{pc})$ and a velocity extent of $\left.33 \mathrm{~km} \mathrm{~s}^{-1}\right)$.

\subsection{PN abundances}

The extinction, determined from the $\mathrm{H} \alpha / \mathrm{H} \beta$ ratio and plotted against galactocentric radius in Fig. 2b, was employed to reddening correct the line fluxes. The values are generally higher than the Galactic extinction along the line of sight to NGC 5128 and indicate either reddening within the early-type galaxy, or local to the nebulae, or both. Ionic abundances can only be computed for a small number of PNe, based on the direct measurements of the [O III]4363A line. Jacoby \& Ciardullo (1999) dealt with the problem of determining PN abundances of extra-galactic PNe (in M 31), where detection of faint diagnostic lines is difficult and expensive in terms of telescope time, by modelling the spectral lines with the Cloudy photoionization code (Ferland et al. 1998); see also Magrini et al. (2004) for application to PN in M33. The simplest nebular model was considered in the absence of further data: a spherical shell of constant density and a black body central star. Line fluxes of $40 \mathrm{PN}$ were matched (considering line flux errors) by Cloudy models to determine abundances of $\mathrm{He}, \mathrm{N}, \mathrm{O}, \mathrm{Ne}$, and, when their lines were detected, 
Ar and $\mathrm{S}$, and $L$ and $T_{\text {eff }}$ of the central stars. Full details are given in Walsh et al. in prep.

The range of $12+\mathrm{O} / \mathrm{H}$ determined from the photoionization models is 8.1 to 9.0 (i.e. -0.7 to +0.3 Solar) with no obvious radial gradient out to $19 \mathrm{kpc}$; the mean $[\mathrm{O} / \mathrm{H}]$ is less than Solar, comparable to the value for the LMC (Leisy \& Dennefeld (2006). O/H can generally be determined to a repeatability of 0.15 dex or better depending on the quality of the spectrum and the temperature of the central star. The highest PN abundances are found closest to the galaxy centre; in this region abundances in H II regions in the dust lane are suggested to be high $([\mathrm{O} / \mathrm{H}] \sim+0.2$, Möllenhoff $(1981))$ and additional FORS observations have been obtained to measure higher quality spectra.

Along each slitlet the stellar continuum can also be measured and from Lick indices of $\mathrm{H} \beta, \mathrm{Mg} \mathrm{b}$ and Fe I absorption lines, the metallicity of the underlying stellar continuum in the vicinity of the PNe can be determined. Comparison to stellar evolutionary tracks, enables the stellar metallicity to be compared to the $\mathrm{O}$ abundance of the PNe. Assuming that both stars and $\mathrm{PNe}$ are co-eval, then $[\mathrm{O} / \mathrm{Fe}]$ can be used as a probe of the star formation history.

Photometric studies indicate that the bulk of stars ( 75\%) in NGC 5128 formed $\sim 12$ Gyr ago, while the minor component ( $25 \%$ ) may be as young as 2-4 Gyr (Rejkuba et al. 2011). Comparison with Marigo et al. (2004) models shows that the brightest PNe correspond to progenitor stars of at least $\sim 2 M_{\odot}$, which implies that the brightest PNe may belong to the young stellar component, or they belong to the old population, and a binary scenario is required to account for their high luminosity (c.f. Ciardullo 2010).

\section{References}

Abdo, A. A., Ackermann, M., Ajello, M., et al. 2010, ApJ, 719, 1433

Ciardullo, R. 2010, PASA, 27, 149

Cortesi, A., Merrifield, M. R., Arnaboldi, M., et al. 2011, MNRAS, 414, 642

Ferland, G. J., Korista, K. T., Verner, D. A., et al. 1998, PASP, 404, 166

Graham, J. A. 1979, ApJ, 232, 60

Harris, G. L. H., Rejkuba, M., \& Harris, W. E. 2010, PASA, 27, 457

Hui, X., Ford, H. C., Ciardullo, R., et al. 1993, ApJS, 88, 423

Hui, X., Ford, H. C., Freeman, K. C., et al. 1995, ApJ, 449, 592

Isreal, F. P. 1998, $A R A A, 8,237$

Jacoby, G. H. \& Ciardullo, R. 1999, ApJ, 515, 169

Kraft, R. P., Forman, W. R., Jones, C., et al. 2002, ApJ, 569, 54

Leisy, P. Dennefeld, M. 2006, A\&GA, 456, 451

Magrini, L., Perinotto, M., Mampaso, A., et al. 2004, A\&A, 426, 779

Malin, D. F., Quinn, P., \& Graham, J. 1983, ApJ, 272, L5

Marigo, P., Girardi, L., Weiss, A., et al. 2004, A\& $A$, 423, 995

Möllenhoff, C. 1981, A\&A, 99, 341

Neumayer, N. 2010, PASA, 27, 449

Peng, E. W., Ford, H. C., Freeman, K. C., et al. 2002, AJ, 124, 3144

Peng, E. W., Ford, H. C., \& Freeman, K. C. 2004, ApJ, 602, 705

Rejkuba, M., Harris, W. E., Greggio, L., et al. 2011, A\&\&A, 526, 123

Sarzi, M., Falcón-Barroso, J., Davies, R. L., et al. 2006, MNRAS, 366, 1151

Steinle, H. 2010, PASA, 27, 431

Woodley, K., A., Harris, W. E., Puzia, T. H., et al. 2010, ApJ, 708, 1335

Woodley, K., A. \& Harris, W. E. 2011, AJ, 708, 1335 